\title{
COMPLEX LAPLACIANS ON ALMOST-HERMITIAN MANIFOLDS
}

\author{
CHUAN-CHIH HSIUNG \& JOHN J. LEVKO III
}

\section{Introduction}

In [2] Hsiung (i) defined a new complex Laplacian $\square_{2}$ for an almostHermitian structure, which is different from the one, denoted by $\square_{1}$, given by Kodaira and Spencer [3], (ii) verified for $\square_{2}$ the well-known conjecture that if $\square_{2}=\Delta / 2$ for all 0 - and 1 -forms, where $\Delta$ is the real Laplacian, then the structure is Kählerian, (iii) studied the conditions for $\square_{2}$ to be real for all 0and 1-forms. Very recently, Ogawa [5] continued Hsiung's work to show that if either $\square_{2}$ or $\square_{1}$ is real for all 0 - and 1-forms, then the structure is Kählerian.

The purpose of this paper is to introduce three more complex Laplacians $\square_{3}$, $\square_{4}, \square_{5}$ for an almost-Hermitian structure and to study the conditions for these Laplacians to be real, together with some relationships among all $\square$ 's. We shall continue to use Hsiung's method [2] which is somewhat different from Ogawa's, and also for completeness we shall reprove Ogawa's result here.

$\S 1$ contains fundamental notation and real operators on a Riemannian manifold. In $\S 2$ we define various almost-Hermitian structures first and then some complex operators for an almost Hermitian structure leading to the complex Laplacians $\square_{i}, i=1, \cdots, 5$. Some conditions for the tensor of an almostHermitian structure to be Kählerian are also given for use in the proofs of our main theorems. $\S 3$ is devoted to the computation of $\square_{i} \xi$ and $\square_{i} \eta, i=1, \cdots$, 5 , for any 0 -form $\xi$ and 1 -form $\eta$ on an almost-Hermitian manifold. In $\S 4$ we show that for an almost-Hermitian structure if the complex Laplacian $\square_{i}$, $i=1,2$ or 4 is real with respect to all 0 - and 1 -forms, then the structure is Kählerian. In $\S 5$ we obtain the following relationships among the $\square$ 's: If for an almost-Hermitian structure the relation $\operatorname{Im} \square_{1}=\operatorname{Im} \square_{i}(i=2$ or 4$)$ or $\operatorname{Im} \square_{2}=\operatorname{Im} \square_{j}(j=4$ or 5) holds for all 0 - and 1-forms, where Im denotes the imaginary part, then the structure is Kählerian.

Throughout this paper, the dimension of a manifold $M^{n}$ is understood to be $n \geq 2$, and all forms and structures are of class at least $C^{2}$.

Communicated July 29, 1970. Research partially supported by the National Science Foundation grant GP-11965. 


\section{Notation and real operators}

Let $M^{n}$ be a Riemannian manifold of dimension $n(\geq 2),\left\|g_{i j}\right\|$ with $g_{i j}=g_{j i}$ be the matrix of the positive definite metric of $M^{n}$, and $\left\|g^{i j}\right\|$ be the inverse matrix of $\left\|g_{i j}\right\|$. Throughout this paper all Latin indices take the values $1, \cdots, n$ unless stated otherwise. We shall follow the usual tensor convention that indices can be raised and lowered by using $g^{i j}$ and $g_{i j}$ respectively, and also that when a Latin letter appears in any term as a subscript and superscript, it is understood that this letter is summed over its range. Moreover, if we multiply, for example, the components $a_{i j}$ of a tensor of type $(0,2)$ by the components $b^{j k}$ of a tensor of type $(2,0)$, it will always be understood that $j$ is to be summed.

Let $\mathcal{N}$ be the set $\{1, \cdots, n\}$ of positive integers less than or equal to $n$, and let $I(p)$ denote an ordered subset $\left\{i_{1}, \cdots, i_{p}\right\}$ of the set $\mathscr{N}$ for $p \leq n$. If the elements $i_{1}, \cdots, i_{p}$ are in the natural order, that is, if $i_{1}<\ldots<i_{p}$, then the ordered set $I(p)$ is denoted by $I_{0}(p)$. Furthermore, denote the nondecreasingly ordered $p$ tuple having the same elements as $I(p)$ by $\langle I(p)\rangle$, and let $I(p ; \hat{s} \mid j)$ be the ordered set $I(p)$ with the $s$-th element $i_{s}$ replaced by another element $j$ of $\mathscr{N}$, which may or may not belong to $I(p)$. We shall use these notations for indices throughout this paper. When more than one set of indices is needed at one time, we may use other capital letters such as $J, K, L, \ldots$ in addition to $I$.

At first we define

$$
\varepsilon_{K(p)}^{J(p)}=\left\{\begin{array}{lc}
0, & \text { if }\langle J(p)\rangle \neq\langle K(p)\rangle \\
0, & \text { if } J(p) \text { or } K(p) \text { contains repeated integers, } \\
+1 \text { or }-1, & \text { if the permutation taking } J(p) \text { into } K(p) \text { is } \\
& \text { even or odd. }
\end{array}\right.
$$

By counting the number of terms it is easy to verify that

$$
\begin{gathered}
\varepsilon_{1}^{I(p) J(n-p)} \varepsilon_{I(p) K(n-p)}^{1 \cdots n}=p ! \varepsilon_{K(n-p)}^{J(n-p)}, \\
\varepsilon_{K(p+q)}^{I(p) J(q)} \varepsilon_{I(p)}^{L(p)}=p ! \varepsilon_{K(p+q)}^{L(p) J(q)} .
\end{gathered}
$$

On the manifold $M^{n}$, let $\nabla$ denote the covariant derivation with respect to the affine connection $\Gamma$, with components $\Gamma_{j k}^{i}$ in local coordinates $x^{1}, \cdots, x^{n}$, of the Riemannian metric $g$, and let $\phi$ be a differential form of degree $p$ given by

$$
\phi=\frac{1}{p !} \phi_{I(p)} d x^{I(p)}=\phi_{I_{0}(p)} d x^{I_{0}(p)}
$$

where $\phi_{I(p)}$ is a skew-symmetric tensor of type $(0, p)$, and we have placed

$$
d x^{I(p)}=d x^{i_{1}} \wedge \cdots \wedge d x^{i_{p}} .
$$

Then we have 


$$
d \phi=(d \phi)_{I_{0}(p+1)} d x^{I_{0}(p+1)},
$$

where

$$
(d \phi)_{I(p+1)}=\frac{1}{p !} \varepsilon_{I(p+1)}^{k J(p)} \nabla_{k} \phi_{J(p)} .
$$

Denote

$$
e_{I(n)}=\varepsilon_{I(n)}^{1 \cdots n}\left(\operatorname{det}\left(g_{i j}\right)\right)^{1 / 2} .
$$

Then by using orthonormal local coordinates $x^{1}, \ldots, x^{n}$ and relation (1.2) we can easily obtain

$$
e_{I(p) K(n-p)} e^{I(p) J(n-p)}=p ! \varepsilon_{K(n-p)}^{J(n-p)} .
$$

The dual operator $*$ is defined by (see, for instance, [6])

$$
* \phi=(* \phi)_{I_{0}(n-p)} d x^{I_{0}(n-p)},
$$

where

$$
(* \phi)_{I(n-p)}=\frac{1}{p !} e_{J(p) I(n-p)} \phi^{J(p)} .
$$

From (1.10), (1.11) it follows that for the scalar 1

$$
* 1=\left(\operatorname{det}\left(g_{i j}\right)\right)^{1 / 2} d x^{1} \wedge \cdots \wedge d x^{n},
$$

which is just the element of area of the manifold $M^{n}$. By using orthonormal local coordinates $x^{1}, \cdots, x^{n}$ we can easily verify that

$$
* * \phi=(-1)^{p(n-p)} \phi .
$$

Denote the inverse operator of $*$ by $*^{-1}$. Then from (1.13) it is seen that on forms of degree $p$

$$
*^{-1}=(-1)^{p(n-p)} * .
$$

The codifferential operator $\delta$ is defined by

$$
\delta \phi=(-1)^{p+n+1} *^{-1} d * \phi .
$$

Making use of (1.6), (1.7), (1.10), (1.11) we obtain immediately

$$
\delta \phi=(\delta \phi)_{I_{0}(p-1)} d x^{I_{0}(p-1)},
$$

where 


$$
(\delta \phi)_{I(p-1)}=-\nabla_{j} \phi_{I(p-1)}^{j} .
$$

For a form $\phi$ of degree $p$ defined by (1.4) we can obtain

$$
\begin{aligned}
p !(\Delta \phi)_{I(p)}= & -\nabla^{j} \nabla_{j} \phi_{I(p)}+\sum_{s=1}^{p} \phi_{I(p ; \hat{s} \mid a)} R^{a}{ }_{i_{s}} \\
& +\sum_{s<t}^{1, \cdots, p} \phi_{I(\boldsymbol{p} ; \hat{s}|a, \hat{t}| b)} R^{a b}{ }_{i_{s} i_{t}},
\end{aligned}
$$

where $\Delta$ is the Laplace-Beltrami operator defined by

$$
\Delta=\delta d+d \delta
$$

and

$$
\begin{gathered}
\nabla^{j}=g^{j k} \nabla_{k}, \\
R_{j k l}^{i}=\partial \Gamma_{j k}^{i} / \partial x^{l}-\partial \Gamma_{j l}^{i} / \partial x^{k}+\Gamma_{j k}^{h} \Gamma_{h l}^{i}-\Gamma_{j l}^{h} \Gamma_{h k}^{i}, \\
R_{j k}=R_{j k s}^{s} .
\end{gathered}
$$

\section{Complex structures and operators}

On a Riemannian manifold $M^{n}$ with metric tensor $g_{i j}$, if there exists a tensor $F_{i}{ }^{j}$ of type $(1,1)$ satisfying

$$
F_{i}{ }^{j} F_{j}{ }^{k}=-\varepsilon_{i}^{k},
$$

then $F_{i}{ }^{j}$ is said to define an almost-complex structure on the manifold $M^{n}$, and the manifold $M^{n}$ is called an almost-complex manifold. From (2.1) it follows that the almost-complex structure $F_{i}{ }^{j}$ induces an automorphism $J$ of the tangent space of the manifold $M^{n}$ at each point with $J^{2}=-I, I$ being the identity operator, such that, for any tangent vector $v^{k}$,

$$
J: v^{k} \rightarrow F_{i}{ }^{k} v^{i}
$$

If an almost-complex structure $F_{i}{ }^{j}$ further satisfies

$$
g_{i j} F_{h}{ }^{i} F_{k}^{j}=g_{h k},
$$

then $F_{i}{ }^{j}$ is said to define an almost-Hermitian structure on the manifold $M^{n}$, and the manifold $M^{n}$ is called an almost-Hermitian manifold. From (2.1), (2.3) it follows that the tensor $F_{i j}$ of type $(0,2)$ defined by

$$
F_{i j}=g_{j k} F_{i}{ }^{k}
$$

is skew-symmetric. Thus on an almost-Hermitian manifold we have the associated differential form 


$$
\omega=F_{i j} d x^{i} \wedge d x^{j} .
$$

By using the multiplication of matrices, from (2.1) we readily see that a necessary condition for the existence of an almost-complex structure on a Riemannian manifold $M^{n}$ is that the dimension $n$ of the manifold $M^{n}$ be even. It should also be remarked that an almost-complex manifold is always orientable, and the orientation depends only on the tensor $F_{i}{ }^{j}$.

An almost-Hermitian structure $F_{i}{ }^{j}$ defined on a manifold $M^{n}$ is called an almost-Kählerian structure and the manifold $M^{n}$ an almost-Kählerian manifold, if the associated form $\omega$ is closed, that is,

$$
d \omega=0 .
$$

From (2.5), (2.6) it follows that an almost-Kählerian structure $F_{i}{ }^{j}$ satisfies

$$
F_{h i j} \equiv \nabla_{h} F_{i j}+\nabla_{i} F_{j h}+\nabla_{j} F_{h i}=0 .
$$

The tensor $\boldsymbol{F}_{h i j}$ is obviously skew-symmetric in all indices.

An almost-Hermitian structure $F_{i}{ }^{j}$ (respectively manifold) satisfying

$$
F_{i} \equiv-\nabla_{j} F_{i}{ }^{j}=0
$$

is called an almost-semi-Kählerian structure (respectively manifold). In particular, the structure $F_{i}{ }^{j}$ is Kählerian if $\nabla_{i} F_{j}{ }^{k}=0$. In this case, by means of (2.1) it is easily seen that the torsion tensor

$$
t_{i j}{ }^{k}=F_{j}{ }^{h}\left(\partial F_{i}{ }^{k} / \partial x^{h}-\partial F_{h}{ }^{k} / \partial x^{i}\right)-F_{i}{ }^{h}\left(\partial F_{j}{ }^{k} / \partial x^{h}-\partial F_{h}{ }^{k} / \partial x^{j}\right)
$$

vanishes, so that the integrability condition of the almost-complex structure $F_{i}{ }^{j}$ is satisfied. But in general when $t_{i j}{ }^{k}=0$, the almost-Hermitian structure $F_{i}{ }^{j}$ is defined to be Hermitian

Multiplying (2.4) by $F^{h i}$ we obtain

$$
F_{i j} F^{h i}=-\varepsilon_{j}^{h} .
$$

By taking covariant differentiation of both sides of (2.9), noticing that

$$
F^{i j} \nabla_{h} F_{i j}=0,
$$

and making use of (2.7), (2.8) it is easily seen that

$$
F_{h i j} F^{i j}=2 F_{h}^{i} F_{i} .
$$

Thus an almost-semi-Kählerian structure $F_{i}{ }^{j}$ satisfies

$$
F_{h i j} F^{i j}=0 \text {. }
$$


Multiplication of (2.11) by $F_{k}{ }^{h}$ and use of (2.9) give

$$
F_{k}=-\frac{1}{2} F_{h i j} F^{i j} F_{k}{ }^{h} .
$$

From (2.7), (2.8), (2.13) we hence conclude that an almost-Kählerian structure or manifold is also almost-semi-Kählerian.

In the proofs of our theorems we shall need the following lemmas.

Lemma 2.1. An almost-Hermitian structure $F$ satisfying

$$
\nabla_{i} F_{j k}=\nabla_{j} F_{i k}
$$

is Kählerian.

Proof. From the skew-symmetry of $F_{i j}$ we have

$$
\nabla_{i} F_{j k}+\nabla_{i} F_{k j}=0 \text {. }
$$

Taking the sum of (2.15) and the two similar equations obtained from it by cyclic permutation of the indices $i, j, k$, and making use of (2.14) we obtain $\nabla_{i} F_{j k}+\nabla_{i} F_{k j}+\nabla_{j} F_{k i}=0$, which together with (2.15) implies immediately $\nabla_{j} F_{k i}=0$.

Lemma 2.2. An almost-Hermitian structure $F$ satisfying

$$
F^{i j} \nabla^{k} \nabla_{k} F_{i j}=0
$$

is Kählerian.

Proof. From (2.9) we have

$$
0=\nabla^{k} \nabla_{k}\left(F_{i j} F^{i j}\right)=2\left(F^{i j} \nabla^{k} \nabla_{k} F_{i j}+\nabla_{k} F_{i j} \nabla^{k} F^{i j}\right)
$$

which together with (2.16) gives $\nabla_{k} F_{i j} \nabla^{k} F^{i j}=0$ and therefore $\nabla_{k} F_{i j}=0$.

Lemma 2.3 ( $S$. Kotô [4]). An almost-Hermitian structure $F$ satisfying

$$
\begin{array}{r}
\nabla_{i} F_{j}{ }^{k}+\nabla_{j} F_{i}{ }^{k}=0, \\
R_{h i}=-\frac{1}{2} R_{h j k l} F^{k l} F_{i}{ }^{j}
\end{array}
$$

is Kählerian.

Proof. (2.17) can be written as

$$
\nabla_{i} F_{j k}=\nabla_{k} F_{i j}
$$

Multiplying (2.19) by $F^{i j}$, using (2.10) and taking the covariant derivative $\nabla_{l}$ of the resulting equation, we obtain, in consequence of (2.19),

$$
F^{i j} \nabla_{l} \nabla_{i} F_{j k}+\nabla_{k} F_{i j} \nabla_{l} F^{i j}=0 .
$$


On the other hand, using (2.19) and the relation $-F^{i j} \nabla_{j} \nabla_{i} F_{k l}=F^{i j} \nabla_{i} \nabla_{j} F_{k l}$, from the Ricci identity it follows respectively that

$$
\begin{aligned}
& \nabla_{l} \nabla_{i} F_{j k}=\nabla_{i} \nabla_{j} F_{k l}+R_{a j i l} F_{k}{ }^{a}-R_{a k i l} F_{j}{ }^{a}, \\
& F^{i j} \nabla_{i} \nabla_{j} F_{k l}=-\frac{1}{2} F^{i j}\left(R_{k j i}^{a} F_{a l}+R^{a}{ }_{l j i} F_{k a}\right) .
\end{aligned}
$$

Similarly, the Bianchi identity leads to

$$
\begin{aligned}
2 R_{h i j k} F^{i j} & =R_{h i j k} F^{i j}-R_{h j i k} F^{i j} \\
& =\left(R_{h j i k}+R_{h i k j}\right) F^{i j}-2 R_{h k i j} F^{i j},
\end{aligned}
$$

and therefore to

$$
R_{h i j k} F^{i j}=-\frac{1}{2} R_{h k i j} F^{i j}
$$

Substituting (2.21) in (2.20) and using (2.22), (2.23), (2.1) we can obtain

$$
\nabla_{k} F_{i j} \nabla_{l} F^{i j}=R_{k l}+F^{i j}\left(\frac{1}{2} R_{a k i j} F_{l}^{a}-R_{a l i j} F_{k}^{a}\right) .
$$

Interchanging $k, l$ in (2.24) and subtracting the resulting equation from (2.24) we have

$$
R_{a k i j} F_{l}^{a} F^{i j}=R_{a l i j} F_{k}^{a} F^{i j},
$$

and therefore (2.24) is reduced to

$$
\nabla_{k} F_{i j} \nabla_{l} F^{i j}=R_{k l}-\frac{1}{2} R_{a l i j} F_{k}^{a} F^{i j}
$$

which together with (2.18) implies

$$
\nabla_{k} F_{i j} \nabla_{l} F^{i j}=0
$$

Multiplying (2.27) by $g^{k l}$ we hence obtain $\nabla_{k} F_{i j}=0$.

Lemma 2.4. For an almost-Hermitian structure $F$, condition

$$
F_{i}{ }^{k} R_{j k}=F_{k}{ }^{l} R^{k}{ }_{i j l}
$$

implies condition (2.18).

Proof. Since

$$
\begin{aligned}
F^{k l} R_{k j h l} & =\frac{1}{2} F^{k l}\left(R_{k j h l}-R_{l j h k}\right) \\
& =\frac{1}{2} F^{k l}\left(R_{k j h l}+R_{k h l j}\right)=\frac{1}{2} F^{k l} R_{k l j h}
\end{aligned}
$$


by the Bianchi identity, from (2.28) we obtain

$$
F_{j}^{k} R_{h k}=\frac{1}{2} R_{j h k l} F^{k l}
$$

Multiplying (2.29) by $F_{i}{ }^{j}$ and using (2.1) lead immediately to (2.18).

We now consider an almost-Hermitian manifold $M^{n}$ with an almost-Hermitian structure $F$, and shall follow Spencer (compare [7, Chapter IX]) to introduce complex operators on the manifold $M^{n}$. At first we define

$$
\prod_{1,0} i^{j}=\frac{1}{2}\left(\varepsilon_{i}{ }^{j}-\sqrt{-1} F_{i}{ }^{j}\right)
$$

and its conjugate ${ }^{1}$ tensor

$$
\prod_{0,1} i^{j}=\prod_{1,0} i^{j}=\frac{1}{2}\left(\varepsilon_{i}^{j}+\sqrt{-1} F_{i}{ }^{j}\right)
$$

A simple calculation gives the following identities:

$$
\begin{aligned}
& \prod_{1,0} i^{j} \prod_{1,0} j^{k}=\prod_{1,0} i^{k}, \\
& \prod_{1,0} i^{j} \prod_{0,1} j^{k}=0, \\
& \prod_{0,1} i^{j} \prod_{0,1} j^{k}=\prod_{0,1} i^{k} .
\end{aligned}
$$

Let $\rho+\sigma=p, \rho \geq 0, \sigma \geq 0$, set

$$
\begin{aligned}
& \prod_{\rho, \sigma} I(p) J(p)=\varepsilon_{I(p)}^{M(\rho) N(\sigma)} \prod_{1,0} m_{1}^{r_{1}} \cdots \prod_{1,0} m_{\rho}{ }^{r_{\rho}} \\
& \cdot \prod_{0,1} n_{1}^{s_{1}} \cdots \prod_{0,1} n_{\sigma}{ }^{s_{\sigma}} \varepsilon_{R_{0}(\rho) S_{0}(\sigma)}^{J(p)},
\end{aligned}
$$

and define $\prod_{\rho, \sigma} I(p){ }^{J(p)}$ to be the identity for $\rho=\sigma=0$ and to be zero for either $\rho<0$ or $\sigma<0$. Then for a form $\phi$ given by (1.4) we have

$$
\prod_{\rho, \sigma} \phi=\left(\prod_{\rho, \sigma} \phi\right)_{I_{0}(p)} d x^{I_{0}(p)}
$$

where

$$
\left(\prod_{\rho, \sigma} \phi\right)_{I(p)}=\prod_{\rho, \sigma}{ }_{I(p)}^{J_{0}(p)} \phi_{J_{0}(p)} .
$$

We next define a complex covariant differentiator

\footnotetext{
1 Throughout this paper a bar over a letter or symbol denotes the conjugate of the complex number or operator defined by the letter or symbol.
} 


$$
\mathscr{D}_{i}=\prod_{1,0}{ }_{i}^{j} \nabla_{j}
$$

and the corresponding contravariant differentiator

$$
\mathscr{D}^{i}=g^{i k} \mathscr{D}_{k}=\prod_{0,1}{ }_{j}{ }^{i} \nabla^{j}=\bar{\prod}_{1,0}{ }_{j}{ }^{i} \nabla^{j} .
$$

The conjugate operators of $\mathscr{D}_{i}$ and $\mathscr{D}^{i}$ are

$$
\begin{aligned}
& \overline{\mathscr{D}}_{i}=\prod_{0,1}{ }^{j} \nabla_{j}, \\
& \overline{\mathscr{D}}^{i}=\prod_{1,0}{ }_{j}{ }^{i} \nabla^{j} .
\end{aligned}
$$

Now we define the complex analogues of the real operators $d$ and $\delta$ defined by (1.7), (1.15) respectively:

$$
\begin{aligned}
d_{1} & =\sum_{\rho+\sigma=p} \prod_{\rho+1, \sigma} d \prod_{\rho, \sigma}, \\
d_{2} & =\sum_{\rho+\sigma=\rho} \prod_{\rho+2, \sigma-1} d \prod_{\rho, \sigma}, \\
\delta_{1} & =\sum_{\rho+\sigma=p} \prod_{\rho, \sigma-1} \delta \prod_{\rho, \sigma}, \\
\delta_{2} & =\sum_{\rho+\sigma=p} \prod_{\rho+1, \sigma-2} \delta \prod_{\rho, \sigma} .
\end{aligned}
$$

The conjugate operators of $d_{1}, d_{2}$ and $\delta_{1}, \delta_{2}$ have the forms:

$$
\begin{aligned}
& \bar{d}_{1}=\sum_{\rho+\sigma=p} \prod_{\rho, \sigma+1} d \prod_{\rho, \sigma}, \\
& \bar{d}_{2}=\sum_{\rho+\sigma=p} \prod_{\rho-1, \sigma+2} d \prod_{\rho, \sigma}, \\
& \bar{\delta}_{1}=\sum_{\rho+\sigma=p} \prod_{\rho-1, \sigma} \delta \prod_{\rho, \sigma}, \\
& \bar{\delta}_{2}=\sum_{\rho+\sigma=p} \prod_{\rho-2, \sigma+1} \delta \prod_{\rho, \sigma} .
\end{aligned}
$$

Furthermore, for a $p$-form $\phi$ given by (1.4) we define

$$
\begin{aligned}
\left(\partial_{1} \phi\right)_{I(p+1)} & =\left(2 d_{2}+d_{1}-\bar{d}_{2}\right)_{I(p+1)}, \\
\left(\vartheta_{1} \phi\right)_{I(p-1)} & =\left(2 \delta_{2}+\delta_{1}-\bar{\delta}_{2}\right)_{I(p-1)}, \\
\left(\partial_{2} \phi\right)_{I(p+1)} & =\sum_{\rho+\sigma=p} \prod_{\rho+1, \sigma} I_{(p+1)}{ }^{j J_{0}(p)} \mathscr{D}_{j} \phi_{J_{0}(p)}, \\
\left(\vartheta_{2} \phi\right)_{I(p-1)} & =-\sum_{\rho+\sigma=p} \prod_{\rho, \sigma} i_{I(p-1)}{ }^{J_{0}(p)} \mathscr{D}^{i} \phi_{J_{0}(p)},
\end{aligned}
$$

together with their conjugate operators: 


$$
\begin{aligned}
&\left(\partial_{1} \phi\right)_{I(p+1)}=\left(2 \bar{d}_{2}+\bar{d}_{1}-d_{2}\right)_{I(p+i)}, \\
&\left(\bar{\vartheta}_{1} \phi\right)_{I(p-1)}=\left(2 \bar{\delta}_{2}+\bar{\delta}_{1}-\delta_{2}\right)_{I(p-1)}, \\
&\left(\bar{\partial}_{2} \phi\right)_{I(p+1)}=\sum_{\rho+\sigma=p} \prod_{\rho, \sigma+1} I_{(p+1)}^{j J_{0}(p)} \bar{\delta}_{j} \phi_{J_{0}(p)}, \\
&\left(\bar{\vartheta}_{2} \phi\right)_{I(p-1)}=-\sum_{\rho+\sigma=p} \prod_{\rho, \sigma} i I(p-1) \\
& J_{J}(p) \overline{\mathscr{D}}^{i} \phi_{J_{0}(p)}
\end{aligned}
$$

It is known that (see [3], [5])

$$
\vartheta_{1}=-* \partial_{1} *, \quad \vartheta_{2}=-* \partial_{2} *,
$$

and that (see [3]) if the structure $F$ of the manifold $M^{n}$ is Kählerian, then $\bar{d}_{2} \phi=d_{2} \phi=0$ for any form $\phi$, and therefore $\partial_{1}=d_{1}$.

Now we introduce the following complex Laplace-Beltrami operators:

$$
\begin{aligned}
& \square_{i}=\bar{\vartheta}_{i} \partial_{i}+\partial_{i} \bar{\vartheta}_{i}, \quad(i=1,2), \\
& \square_{3}=\bar{\vartheta}_{1} \partial_{2}+\partial_{2} \bar{\vartheta}_{1}, \\
& \square_{4}=\bar{\vartheta}_{2} \partial_{1}+\partial_{1} \bar{\vartheta}_{2}, \\
& \square_{5}=\bar{\delta}_{1} d_{1}+d_{1} \bar{\delta}_{1} .
\end{aligned}
$$

It should be noted that $\square_{1}$ was first defined by Kodaira-Spencer [3], and $\square_{2}$ by Hsiung [2].

From [3] we know that $d=\partial_{1}+\bar{\partial}_{1}$. In order to apply $\partial_{2}+\bar{\partial}_{2}$, let $\xi$ be any 0 -form. The we have, in consequence of (2.50), (2.36), (2.32), (2.30),

$$
\left(\partial_{2} \xi\right)_{i_{1}}=\prod_{1,0} i_{1}^{j} \nabla_{j} \xi=\frac{1}{2}\left(\nabla_{i_{1}} \xi-\sqrt{-1} F_{i_{1}}{ }^{j} \nabla_{j} \xi\right)
$$

which together with (1.6), (1.7) gives

$$
d \xi=\left(\partial_{2}+\bar{\partial}_{2}\right) \xi \text {. }
$$

Similarly, for any 1 -form $\eta$, using $(2.50),(2.36),(2.33),(2.34),(2.35),(2.32)$ we can obtain

$$
\begin{aligned}
\left(\partial_{2} \eta\right)_{i_{1} i_{2}} & =\prod_{1,0} i_{1}{ }^{j} \prod_{1,0} i_{2}{ }^{k}\left(\nabla_{j} \eta_{k}-\nabla_{k} \eta_{j}\right)+\left(\prod_{1,0} i^{j}{ }^{j} \prod_{0,1} i_{2}{ }^{k}-\prod_{1,0} i_{2}{ }^{j} \prod_{0,1}{ }^{k}{ }^{k}\right) \nabla_{j} \eta_{k} \\
& =\frac{1}{2}\left[\nabla_{i_{1}} \eta_{i_{2}}-\nabla_{i_{2}} \eta_{i_{1}}+\sqrt{-1}\left(F_{i_{2}}{ }^{j} \nabla_{j} \eta_{i_{1}}-F_{i_{1}}{ }^{j} \nabla_{j} \eta_{i_{2}}\right)\right]
\end{aligned}
$$

which together with (1.6), (1.7) gives

$$
d \eta=\frac{1}{2}\left(\partial_{2}+\bar{\partial}_{2}\right) \eta \text {. }
$$


The almost-complex structure $F$ of the manifold $M^{n}$ is said [3] to be (completely) integrable if and only if $\partial_{1}^{2}=0$. Now by means of $(2.61),(2.50)$, $(2.30), \cdots,(2.36)$ and the relation

$$
\nabla_{i} \nabla_{j} \xi=\nabla_{j} \nabla_{i} \xi
$$

for any 0 -form $\xi$, an elementary but lengthy calculation gives

$$
\begin{aligned}
4\left(\partial_{2}^{2} \xi\right)_{i_{1} i_{2}}= & \left(F_{i_{2}}{ }^{j} \nabla_{j} F_{i_{1}}{ }^{k}-F_{i_{1}}{ }^{j} \nabla_{j} F_{i_{2}}{ }^{k}\right) \nabla_{k} \xi \\
& +\sqrt{-1}\left(\nabla_{i_{2}} F_{i_{1}}{ }^{k}-\nabla_{i_{1}} F_{i_{2}}{ }^{k}\right) V_{k} \xi .
\end{aligned}
$$

If $\partial_{2}^{2}$ is real for any 0 -form $\xi$, then by taking $\xi=x^{i}$ for any arbitary $i$ with respect to any local coordinates $x^{1}, \cdots, x^{n}$, from (2.66) we obtain (2.14), and therefore by Lemma 2.1 the structure $F$ is Kählerian.

\section{Expressions for $\square$ 's}

In this section we shall give expressions for $\square_{i} \xi$ and $\square_{i} \eta$, where $i=1, \ldots, 4$, and $\xi$ and $\eta$ are respectively any 0 - and 1-forms on an almost-Hermitian manifold $M^{n}$ with an almost-Hermitian structure $F$.

3.1. Laplacian $\square_{2}$. In [2, pp. 146-147] we obtained

$$
\begin{aligned}
& 4 \square_{2} \xi=2 \Delta \xi+\nabla^{h} F_{h}{ }^{j}\left(-F_{j}{ }^{k} \nabla_{k} \xi+\sqrt{-1} \nabla_{j} \xi\right), \\
& 4\left(\square_{2} \eta\right)_{i_{1}}=-F_{i}{ }^{j} \nabla^{i} F_{i_{1}}{ }^{h} \nabla_{h} \eta_{j}-F_{j}{ }^{h} \nabla^{i} F_{i}{ }^{j} \nabla_{h} \eta_{i_{1}}+F_{i_{1}}{ }^{i} \nabla_{i} F_{h}{ }^{j} \nabla^{h} \eta_{j} \\
& -2 \nabla^{i} \nabla_{\imath} \eta_{i_{1}}+\left[\nabla_{j}, \nabla_{i_{1}}\right] \eta^{j}+F_{i_{1}}{ }^{h} F^{i j}\left[\nabla_{h}, \nabla_{i}\right] \eta_{j} \\
& +\sqrt{-1}\left\{\nabla^{i} F_{i}{ }^{j} \nabla_{j} \eta_{i_{1}}-\left(\nabla_{j} F_{i_{1}}{ }^{k}+\nabla_{i_{1}} F_{j}{ }^{k}\right) \nabla_{k} \eta^{j}\right. \\
& \left.+2 F_{j}{ }^{k} \nabla^{j} \nabla_{k} \eta_{i_{1}}-F_{i_{1}}{ }^{k}\left[\nabla_{j}, \nabla_{k}\right] \eta^{j}-F^{k j}\left[\nabla_{k}, \nabla_{i_{1}}\right] \eta_{j}\right\}
\end{aligned}
$$

where

$$
\left[\nabla_{h}, \nabla_{i}\right]=\nabla_{h} \nabla_{i}-\nabla_{i} \nabla_{h}
$$

3.2. Laplacian $\square_{1}$. At first we notice that as a result of (2.65) we have

$$
F_{i}{ }^{j} \nabla^{i} \nabla_{j} \xi=0 \text {. }
$$

By using (2.57), (2.53), (2.45), ..., (2.48), (2.33), ((2.40), (2.41), (2.43),(2.1), (2.30), (2.32), (2.34), (2.35), (3.4), (1.17), (1.18) we can obtain

$$
2 \square_{1} \xi=2 \delta \prod_{1,0} d \xi=\Delta \xi+\sqrt{-1} \nabla^{i} F_{i}{ }^{j} \nabla_{j} \xi \text {. }
$$

In order to compute $\square_{1} \eta$, from (2.48), (2.52), (2.40), (2.41), (2.43), (2.45), (2.46), (2.47) we first see that

(3.6) $\partial_{1}=2 \prod_{2,0} d \prod_{0,1}+\prod_{2,0} d \prod_{1,0}+\prod_{1,1} d \prod_{0,1}-\prod_{0,2} d \prod_{1,0}, \quad$ for 1 -forms , 


$$
\overline{\vartheta_{1}}=2 \prod_{0,1} \delta \prod_{2,0}+\prod_{1,0} \delta \prod_{2,0}+\prod_{0,1} \delta \prod_{1,1}-\prod_{1,0} \delta \prod_{0,2}, \quad \text { for } 2 \text {-forms }
$$

Next, by means of (1.6), (1.7), (2.33), (2.34), (2.35), (1.2), (1.3), (2.30), (2.31), we obtain

$$
\begin{aligned}
& \prod_{1,1} d \prod_{0,1} \eta=\left[\prod_{1,0} i_{1}{ }^{k} \prod_{1,0} i_{2}{ }^{l}\left(\nabla_{k} \prod_{0,1} i^{j}-\nabla_{l} \prod_{0,1} k^{j}\right) \eta_{j}+\prod_{1,0} i_{1}{ }^{k} \prod_{0,1} i_{2}{ }^{j} \nabla_{k} \eta_{j}\right. \\
& \left.-\prod_{1,0} i_{2}{ }^{k} \prod_{0,1} i_{1}{ }^{j} \nabla_{k} \eta_{j}+\prod_{1,0} i_{2}{ }^{k} \prod_{0,1} i_{1}{ }^{l}\left(\nabla_{i} \prod_{0,1} k^{j}-\nabla_{k} \prod_{0,1} l^{j}\right) \eta_{j}\right] d x^{I_{0}(2)} \\
& =\frac{1}{4}\left\{\nabla_{i_{1}} \eta_{i_{2}}-\nabla_{i_{2}} \eta_{i_{1}}+F_{i_{1}}{ }^{k} F_{i_{2}}{ }^{j}\left(\nabla_{k} \eta_{j}-\nabla_{j} \eta_{k}\right)\right. \\
& +\sqrt{-1}\left[\eta_{j}\left(\nabla_{i_{1}} F_{i_{2}}{ }^{j}-\nabla_{i_{2}} F_{i_{1}}{ }^{j}+F_{i_{1}}{ }^{k} F_{i_{2}}{ }^{l}\left(\nabla_{k} F_{l}{ }^{j}-\nabla_{l} F_{k}{ }^{j}\right)\right)\right. \\
& \left.\left.+F_{i_{2}}{ }^{j}\left(\nabla_{i_{1}} \eta_{j}+\nabla_{j} \eta_{i_{1}}\right)-F_{i_{1}}{ }^{j}\left(\nabla_{i_{2}} \eta_{j}+\nabla_{j} \eta_{i_{2}}\right)\right]\right\} d x^{I_{0}(2)},
\end{aligned}
$$

$$
\begin{aligned}
& \prod_{2,0} d \prod_{1,0} \eta=\left[\prod_{1,0} i_{1}^{k} \prod_{1,0} i_{2}{ }^{l}\left(\nabla_{k} \prod_{1,0} l^{j}-\nabla_{l} \prod_{1,0} k^{j}\right) \eta_{j}\right. \\
& \left.+\prod_{1,0} i_{1}{ }^{j} \prod_{1,0} i_{2}{ }^{k}\left(\nabla_{j} \eta_{k}-\nabla_{k} \eta_{j}\right)\right] d x^{I_{0}(2)} \\
& =\frac{1}{8}\left\{\eta_{j}\left[F_{i_{1}}{ }^{k}\left(\nabla_{i_{2}} F_{k}{ }^{j}-\nabla_{k} F_{i_{2}}{ }^{j}\right)+{F_{i_{2}}}^{k}\left(\nabla_{k} F_{i_{1}}{ }^{j}-\nabla_{i_{1}} F_{k}{ }^{j}\right)\right]\right. \\
& +2 \nabla_{i_{1}} \eta_{i_{2}}-2 \nabla_{i_{2}} \eta_{i_{1}}+2 F_{i_{1}}{ }^{j} F_{i_{2}}{ }^{k}\left(\nabla_{k} \eta_{j}-\nabla_{j} \eta_{k}\right) \\
& +\sqrt{-1}\left[\eta_{j}\left(\nabla_{i_{2}} F_{i_{1}}{ }^{j}-\nabla_{i_{1}} F_{i_{2}}{ }^{j}+F_{i_{1}}{ }^{k} F_{i_{2}}{ }^{l}\left(\nabla_{k} F_{l}{ }^{j}-\nabla_{l} F_{k}{ }^{j}\right)\right)\right. \\
& \left.\left.+2 F_{i_{1}}{ }^{j}\left(\nabla_{i_{2}} \eta_{j}-\nabla_{j} \eta_{i_{2}}\right)+2 F_{i_{2}}{ }^{j}\left(\nabla_{j} \eta_{i_{1}}-\nabla_{i_{1}} \eta_{j}\right)\right]\right\} d x^{I_{0}(2)},
\end{aligned}
$$

$$
\begin{array}{r}
\prod_{2,0} d \prod_{0,1} \eta=\prod_{1,0}{ }_{i_{1}}{ }^{k} \prod_{1,0} i_{i_{2}}{ }^{l}\left(\nabla_{k} \prod_{0,1} l^{j}-\nabla_{l} \prod_{0,1}{ }^{j}\right) \eta_{j} d x^{I_{0}(2)} \\
=\frac{1}{8}\left\{\eta_{j}\left[F_{i_{1}}{ }^{k}\left(\nabla_{k} F_{i_{2}}{ }^{j}-\nabla_{i_{2}} F_{k}{ }^{j}\right)+F_{i_{2}}{ }^{k}\left(\nabla_{i_{1}} F_{k}{ }^{j}-\nabla_{k} F_{i_{1}}{ }^{j}\right)\right]\right. \\
+\sqrt{-1} \eta_{j}\left[\nabla_{i_{1}} F_{i_{2}}{ }^{j}-\nabla_{i_{2}} F_{i_{1}}{ }^{j}\right. \\
\left.\left.+F_{i_{1}}{ }^{k} F_{i_{2}}{ }^{l}\left(\nabla_{l} F_{k}{ }^{j}-\nabla_{k} F_{l}{ }^{j}\right)\right]\right\} d x^{I_{0}(2)}
\end{array}
$$$$
-\prod_{0,2} d \prod_{1,0} \eta=\prod_{0,1} i_{2}^{k} \prod_{0,1} i_{1}{ }^{l}\left(\nabla_{k} \prod_{1,0} i^{j}-\nabla_{l} \prod_{1,0} k^{j}\right) \eta_{j} d x^{I_{0}(2)}
$$

$$
\begin{gathered}
=\frac{1}{8}\left\{\eta_{j}\left[F_{i_{1}}{ }^{k}\left(\nabla_{i_{2}} F_{k}{ }^{j}-\nabla_{k} F_{i_{2}}{ }^{j}\right)+F_{i_{2}}{ }^{k}\left(\nabla_{k} F_{i_{1}}{ }^{j}-\nabla_{i_{1}} F_{k}{ }^{j}\right)\right]\right. \\
+\sqrt{-1} \eta_{j}\left[\nabla_{i_{1}} F_{i_{2}}{ }^{j}-\nabla_{i_{2}} F_{i_{1}}{ }^{j}\right. \\
\left.\left.+F_{i_{1}}{ }^{k} F_{i_{2}}{ }^{l}\left(\nabla_{l} F_{k}{ }^{j}-\nabla_{k} F_{l}{ }^{j}\right)\right]\right\} d x^{I_{0}(2)}
\end{gathered}
$$

$$
\begin{aligned}
4\left(\prod_{1,0} d \delta \prod_{1,0} \eta\right)_{i_{1}}= & \eta_{j} F_{i_{1}}{ }^{l} \nabla_{l} \nabla^{k} F_{k}{ }^{j}+F_{i_{1}}{ }^{l} \nabla^{k} F_{k}{ }^{j} \nabla_{l} \eta_{j} \\
& +F_{i_{1}}{ }^{l} \nabla_{l} F_{k}{ }^{j} \nabla^{k} \eta_{j}-\nabla_{i_{1}} \nabla^{k} \eta_{k}+F_{i_{1}}{ }^{l} F_{k}{ }^{j} \nabla_{l} \nabla^{k} \eta_{j} \\
& +\sqrt{-1}\left(\eta_{j} \nabla_{i_{1}} \nabla^{k} F_{k}{ }^{j}+\nabla^{k} F_{k}{ }^{j} \nabla_{i_{1}} \eta_{j}+\nabla_{i_{1}} F_{k}{ }^{j} \nabla^{k} \eta_{j}\right. \\
& \left.+F_{k}{ }^{j} \nabla_{i_{1}} \nabla^{k} \eta_{j}+F_{i_{1}}{ }^{l} \nabla_{l} \nabla^{k} \eta_{k}\right) .
\end{aligned}
$$


Substitution of $(3.8), \ldots,(3.11)$ in (3.6) thus gives

$$
\begin{aligned}
\partial_{1} \eta=\frac{1}{2}\left\{\nabla_{i_{1}} \eta_{i_{2}}-\nabla_{i_{2}} \eta_{i_{1}}+\sqrt{-1}\left[\eta_{j}\left(\nabla_{i_{1}} F_{i_{2}}{ }^{j}-\nabla_{i_{2}} F_{i_{1}}{ }^{j}\right)\right.\right. \\
\left.\left.+F_{i_{2}}{ }^{j} \nabla_{j} \eta_{i_{1}}-F_{i_{1}}{ }^{j} \nabla_{j} \eta_{i_{2}}\right]\right\} d x^{I_{0}(2)} .
\end{aligned}
$$

Now put

$$
\begin{aligned}
& A_{i_{1} i_{2}}=\nabla_{i_{1}} \eta_{i_{2}}+\sqrt{-1}\left(\eta_{j} \nabla_{i_{1}} F_{i_{2}}{ }^{j}+F_{i_{2}}{ }^{j} \nabla_{j} \eta_{i_{1}}\right), \\
& \boldsymbol{B}_{s i_{1}}^{k_{1} k_{2}}=\varepsilon_{s}^{k_{1}} \varepsilon_{i_{1}}^{k_{2}}-\varepsilon_{i_{1}}^{k_{1}} \varepsilon_{s}^{k_{2}}+F_{i_{1}}{ }^{k_{1}} F_{s}{ }^{k_{2}}-F_{s}{ }^{k_{1}} F_{i_{1}}{ }^{{ }^{2}{ }_{2}} \text {, } \\
& C_{s i_{1}}^{k_{1} k_{2}}=\varepsilon_{i_{1}}^{k_{1}} F_{s}{ }^{k_{2}}-\varepsilon_{s}^{k_{1}} F_{i_{1}}{ }^{k_{2}}-\varepsilon_{i_{1}}^{k_{2}} F_{s}{ }^{{ }^{k_{1}}}+\varepsilon_{s}{ }^{k_{2}} F_{i_{1}}{ }^{{ }_{1}} \text {. }
\end{aligned}
$$

Then

$$
\partial_{1} \eta=\frac{1}{2}\left(A_{i_{1} i_{2}}-A_{i_{2} i_{1}}\right) d x^{I_{0}(2)} .
$$

By means of (3.13), (2.33), (2.34), (2.35), (2.30), (2.31), (1.16), (1.17), elementary but rather lengthy calculations give

$$
\begin{aligned}
& -8\left(\prod_{0,1} \delta \prod_{1,1} \partial_{1} \eta\right)_{i_{1}} \\
& \left.=2 \prod_{0,1} \nabla^{s}\left[\varepsilon_{s}^{k_{1}} \varepsilon_{i_{1}}^{k_{2}}-\varepsilon_{i_{1}}^{k_{1}} \varepsilon_{s}^{k_{2}}+F_{s}{ }^{k_{1}} F_{i_{1}}{ }^{k_{2}}-F_{i_{1}}{ }^{k_{1}} F_{s}{ }^{k_{2}}\right) A_{k_{1} k_{2}}\right] \\
& =\eta_{j}\left[\nabla^{s} F_{s}{ }^{k}\left(\nabla_{k} F_{i_{1}}{ }^{j}-\nabla_{i_{1}} F_{k}{ }^{j}\right)+F_{i_{1}}{ }^{r} F_{s}{ }^{k} \nabla^{s} F_{r}{ }^{l}\left(\nabla_{l} F_{k}{ }^{j}-\nabla_{k} F_{l}{ }^{j}\right)\right. \\
& \left.+F_{i_{1}}{ }^{r}\left(\nabla^{s} \nabla_{r} F_{s}{ }^{j}-\nabla^{s} \nabla_{s} F_{r}{ }^{j}\right)+F_{s}{ }^{k}\left(\nabla^{s} \nabla_{k} F_{i_{1}}{ }^{j}-\nabla^{s} \nabla_{i_{1}} F_{k}{ }^{j}\right)\right] \\
& +\left(2 F_{i_{1}}{ }^{l} \nabla^{s} F_{s}{ }^{k}-F_{s}{ }^{l} \nabla^{s} F_{i_{1}}{ }^{k}\right) \nabla_{k} \eta_{l}+F_{i_{1}}{ }^{r} \nabla^{s} \eta_{j}\left(\nabla_{r} F_{s}{ }^{j}-2 \nabla_{s} F_{r}{ }^{j}\right) \\
& \text { (3.16) } \quad-F_{s}{ }^{k} \nabla_{i_{1}} F_{k}{ }^{j} \nabla^{s} \eta_{j}-F_{i_{1}}{ }^{r} \nabla^{s} F_{r}{ }^{j} \nabla_{j} \eta_{s}+2 \nabla^{s} \nabla_{s} \eta_{i_{1}} \\
& +2 F_{i_{1}}{ }^{k} F_{s}{ }^{l} \nabla^{s} \nabla_{l} \eta_{k}+\sqrt{-1}\left\{\eta _ { j } \left[F_{s}{ }^{k} \nabla^{s} F_{i_{1}}{ }^{l}\left(\nabla_{k} F_{l}{ }^{j}-\nabla_{l} F_{k}{ }^{j}\right)\right.\right. \\
& +F_{i_{1}}{ }^{k} \nabla^{s} F_{s}{ }^{l}\left(\nabla_{l} F_{k}{ }^{j}-\nabla_{k} F_{l}{ }^{j}\right)+\nabla^{s} \nabla_{s} F_{i_{1}}{ }^{j}-\nabla^{s} \nabla_{i_{1}} F_{s}{ }^{j} \\
& \left.+F_{i_{1}}{ }^{k} F_{s}{ }^{l}\left(\nabla^{s} \nabla_{l} F_{k}{ }^{j}-\nabla^{s} \nabla_{k} F_{l}{ }^{j}\right)\right]+\left(2 \nabla_{s} F_{i_{1}}{ }^{j}-\nabla_{i_{1}} F_{s}{ }^{j}\right) \nabla^{s} \eta_{j} \\
& -2 \nabla^{s} F_{s}{ }^{j} \nabla_{j} \eta_{i_{1}}+\nabla^{s} F_{i_{1}}{ }^{j} \nabla_{j} \eta_{s}-F_{i_{1}}{ }^{k} F_{s}{ }^{l}\left(\nabla_{k} F_{l}{ }^{j} \nabla^{s} \eta_{j}+\nabla^{s} F_{k}{ }^{j} \nabla_{j} \eta_{l}\right) \\
& \left.+2 F_{i_{1}}{ }^{j} \nabla^{s} \nabla_{s} \eta_{j}-2 F_{s}{ }^{j} \nabla^{s} \nabla_{j} \eta_{i_{1}}\right\} \\
& -8\left(\prod_{1,0} \delta \prod_{2,0} \partial_{1} \eta\right)_{i_{1}} \\
& =\prod_{1,0} \nabla^{s}\left[\left(B_{s}^{k_{1} k_{1}}+\sqrt{-1} C_{s i_{1}}^{k_{1} k_{2}}\right) A_{k_{1} k_{2}}\right] \\
& =\eta_{j}\left[\frac{1}{2} \nabla^{s} F_{i_{1}}{ }^{k}\left(\nabla_{s} F_{k}{ }^{j}-\nabla_{k} F_{s}{ }^{j}\right)+\frac{1}{2} F_{i_{1}}{ }^{r} F_{s}{ }^{k} \nabla^{s} F_{r}{ }^{l}\left(\nabla_{l} F_{k}{ }^{j}-\nabla_{k} F_{l}{ }^{j}\right)\right. \\
& +\nabla^{s} F_{s}{ }^{k}\left(\nabla_{k} F_{i_{1}}{ }^{j}-\nabla_{i_{1}} F_{k}{ }^{j}\right)+F_{i_{1}}{ }^{k}\left(\nabla^{s} \nabla_{s} F_{k}{ }^{j}-\nabla^{s} \nabla_{k} F_{s}{ }^{j}\right) \\
& \left.+F_{s}{ }^{k}\left(\nabla^{s} \nabla_{k} F_{i_{1}}{ }^{j}-\nabla^{s} \nabla_{i_{1}} F_{k}{ }^{j}\right)\right]+2 F_{i_{1}}{ }^{k} \nabla^{s} F_{s}{ }^{l}\left(\nabla_{k} \eta_{l}-\nabla_{l} \eta_{k}\right)
\end{aligned}
$$




$$
\begin{aligned}
& +F_{i_{1}}{ }^{r} \nabla_{j} \eta^{s}\left(\nabla_{r} F_{s}{ }^{j}+\nabla_{s} F_{r}{ }^{j}\right)+F_{s}{ }^{k} \nabla^{s} F_{i_{1}}{ }^{l}\left(\nabla_{l} \eta_{k}-2 \nabla_{k} \eta_{l}\right) \\
& -F_{s}{ }^{k} \nabla_{i_{1}} F_{k}{ }^{j} \nabla^{s} \eta_{j}+2 \nabla^{s} \nabla_{s} \eta_{i_{1}}-2 \nabla^{s} \nabla_{i_{1}} \eta_{s} \\
& +2 F_{i_{\mathbf{1}}}{ }^{k} F_{s}{ }^{l}\left(\nabla^{s} \nabla_{k} \eta_{l}-\nabla^{s} \nabla_{l} \eta_{k}\right) \\
& +\sqrt{-1}\left\{\eta _ { j } \left[\frac{1}{2} F_{i_{1}}{ }^{r} \nabla^{s} F_{r}{ }^{k}\left(\nabla_{k} F_{s}{ }^{j}-\nabla_{s} F_{k}{ }^{j}\right)+F_{i_{1}}{ }^{k} \nabla^{s} F_{s}{ }^{l}\left(\nabla_{k} F_{l}{ }^{j}\right.\right.\right. \\
& \left.-\nabla_{l} F_{k}{ }^{j}\right)+\frac{1}{2} F_{s}{ }^{k} \nabla^{s} F_{i_{1}}{ }^{l}\left(\nabla_{l} F_{k}{ }^{j}-\nabla_{k} F_{l}{ }^{j}\right)+\nabla^{s} \nabla_{s} F_{i_{1}}{ }^{j} \\
& \left.-\nabla^{s} \nabla_{i_{1}} F_{s}{ }^{j}+F_{i_{1}}{ }^{k} F_{s}{ }^{l}\left(\nabla^{s} \nabla_{k} F_{l}{ }^{j}-\nabla^{s} \nabla_{l} F_{k}{ }^{j}\right)\right]+\nabla^{s} F_{i_{1}}{ }^{j} \nabla_{j} \eta_{s} \\
& -\nabla_{i_{1}} F_{s}{ }^{j} \nabla^{s} \eta_{j}+F_{i_{1}}{ }^{k} F_{s}{ }^{l} \nabla_{k} F_{l}{ }^{j} \nabla^{s} \eta_{j}-F_{i_{1}}{ }^{r} F_{s}{ }^{l} \nabla^{s} F_{r}{ }^{k} \nabla_{k} \eta_{l} \\
& +2 \nabla^{s} F_{s}{ }^{j}\left(\nabla_{i_{1}} \eta_{j}-\nabla_{j} \eta_{i_{1}}\right)-2 F_{s}{ }^{k} F_{i_{1}}{ }^{l} \nabla_{k} F_{l}{ }^{j} \nabla^{s} \eta_{j} \\
& \left.+2 F_{s}{ }^{k}\left(\nabla^{s} \nabla_{i_{1}} \eta_{k}-\nabla^{s} \nabla_{k} \eta_{i_{1}}\right)+2 F_{i_{1}}{ }^{j}\left(\nabla^{s} \nabla_{j} \eta_{s}-\nabla^{s} \nabla_{s} \eta_{j}\right)\right\} \text {, } \\
& -8\left(\prod_{0,1} \delta \prod_{2,0} \partial_{1} \eta\right)_{i_{1}} \\
& =\eta_{j}\left[\frac{1}{2} \nabla^{s} F_{i_{1}}{ }^{k}\left(\nabla_{s} F_{k}{ }^{j}-\nabla_{k} F_{s}{ }^{j}\right)+\frac{1}{2} F_{i_{1}}{ }^{r} F_{s}{ }^{k} D^{s} F_{r}{ }^{l}\left(\nabla_{k} F_{l}{ }^{j}-\nabla_{l} F_{k}{ }^{j}\right)\right] \\
& +F_{s}{ }^{k} \nabla^{s} F_{i_{1}}{ }^{l}\left(\nabla_{l} \eta_{k}-\nabla_{k} \eta_{l}\right)+F_{i_{1}}{ }^{r} \nabla^{s} F_{r}{ }^{k}\left(\nabla_{s} \eta_{k}-\nabla_{k} \eta_{s}\right) \\
& +\sqrt{-1}\left\{\eta _ { j } \left[\frac{1}{2} F_{s}{ }^{k} \nabla^{s} F_{i_{1}}{ }^{l}\left(\nabla_{l} F_{k}{ }^{j}-\nabla_{k} F_{l}{ }^{j}\right)\right.\right. \\
& \left.+\frac{1}{2} F_{i_{1}}{ }^{r} \nabla^{s} F_{r}{ }^{k}\left(\nabla_{s} F_{k}{ }^{j}-\nabla_{k} F_{s}{ }^{j}\right)\right] \\
& \left.+F_{i_{1}}{ }^{r} F_{s}{ }^{k} \nabla^{s} F_{r}{ }^{l}\left(\nabla_{l} \eta_{k}-\nabla_{k} \eta_{l}\right)+\nabla^{s} F_{i_{1}}{ }^{j}\left(\nabla_{j} \eta_{s}-\nabla_{s} \eta_{j}\right)\right\} \text {, } \\
& -16\left(\prod_{1,0} \delta \prod_{0,2} \partial_{1} \eta\right)_{i_{1}}=2 \prod_{1,0} \nabla^{s}\left[\left(B_{s i_{1}}^{k_{1} k_{2}}-\sqrt{-1} C_{s i_{1}}^{k_{1} k_{2}}\right) A_{k_{1} k_{2}}\right] \\
& =\eta_{j}\left[\nabla^{s} F_{i_{1}}{ }^{k}\left(\nabla_{k} F_{s}{ }^{j}-\nabla_{s} F_{k}{ }^{j}\right)+F_{i_{1}}{ }^{r} F_{s}{ }^{k} \nabla^{s} F_{r}{ }^{l}\left(\nabla_{l} F_{k}{ }^{j}-\nabla_{k} F_{l}{ }^{j}\right)\right] \\
& +\sqrt{-1} \eta_{j}\left[F_{s}{ }^{k} \nabla^{s} F_{i_{1}}{ }^{l}\left(\nabla_{l} F_{k}{ }^{j}-\nabla_{k} F_{l}{ }^{j}\right)\right. \\
& \left.+F_{i_{1}}{ }^{r} \nabla^{s} F_{r}{ }^{k}\left(\nabla_{s} F_{k}{ }^{j}-\nabla_{k} F_{s}^{j}\right)\right] \text {. }
\end{aligned}
$$

Substituting (3.6), (3.7), (3.12), (3.16), .., (3.19) in (2.57) and using (2.32) and

$$
2 F_{j}{ }^{k} \nabla^{j} \nabla_{k} \eta_{i_{1}}=F^{j k}\left[\nabla_{j}, \nabla_{k}\right] \eta_{i_{1}}
$$

we can obtain, after some elementary simplification,

$$
\begin{aligned}
4\left(\square_{1} \eta\right)_{i_{1}}= & 4\left[\left(2 \prod_{0,1} \delta \prod_{2,0}+\prod_{1,0} \delta \prod_{2,0}+\prod_{0,1} \delta \prod_{1,1}-\prod_{1,0} \delta \prod_{0,2}\right) \partial_{1} \eta+\prod_{1,0} d \delta \prod_{1,0} \eta\right]_{i_{x}} \\
= & \eta_{j}\left[\nabla^{s} F_{s}{ }^{k}\left(\nabla_{i_{1}} F_{k}{ }^{j}-\nabla_{k} F_{i_{1}}{ }^{j}\right)+\nabla^{s} F_{i_{1}}{ }^{k}\left(\nabla_{k} F_{s}{ }^{j}-\nabla_{s} F_{k}{ }^{j}\right)\right. \\
& \left.+F_{s}{ }^{k}\left(\nabla^{s} \nabla_{i_{1}} F_{k}{ }^{j}-\nabla^{s} \nabla_{k} F_{i_{1}}{ }^{j}\right)+F_{i_{1}}{ }^{l} \nabla_{l} \nabla^{k} F_{k}{ }^{j}\right] \\
& +F_{s}{ }^{s} \nabla^{s} \eta_{j}\left(\nabla_{i_{1}} F_{k}{ }^{j}-2 \nabla_{k} F_{i_{1}}{ }^{j}\right)+F_{s}{ }^{k} \nabla^{s} F_{i_{1}}{ }^{l}\left(\nabla_{k} \eta_{l}-\nabla_{l} \eta_{k}\right) \\
& +F_{i_{1}}{ }^{l} \nabla_{l} F_{k}{ }^{j} \nabla^{k} \eta_{j}+F_{i_{1}}{ }^{l} \nabla^{j} F_{l}{ }^{k} \nabla_{k} \eta_{j}-2 V^{s} \nabla_{s} \eta_{i_{1}} \\
& +\left[\nabla_{k}, \nabla_{i_{1}}\right] \eta^{k}+F_{i_{1}}{ }^{k} F^{k j}\left[\nabla_{l}, \nabla_{k}\right] \eta_{j} \\
& +\sqrt{-1}\left\{\eta_{j}\left(\nabla^{s} \nabla_{i_{1}} F_{s}{ }^{j}-\nabla^{s} \nabla_{s} F_{i_{1}}{ }^{j}+\nabla_{i_{1}} \nabla^{s} F_{s}{ }^{j}\right)+2 \nabla^{s} F_{s}{ }^{j} \nabla_{j} \eta_{i_{1}}\right.
\end{aligned}
$$




$$
\begin{aligned}
& -2\left(\nabla_{s} F_{i_{1}}{ }^{j}+\nabla_{i_{1}} F_{s}{ }^{j}\right) \nabla_{j} \eta^{s}+F^{s j}\left[\nabla_{s}, \nabla_{j}\right] \eta_{i_{1}} \\
& \left.-F^{s j}\left[\nabla_{s}, \nabla_{i_{1}}\right] \eta_{j}-F_{i_{1}}{ }^{j}\left[\nabla_{s}, \nabla_{j}\right] \eta^{s}\right\} .
\end{aligned}
$$

3.3. Laplacian $\square_{3}$. In the same way as above we can compute $\square_{i} \xi$ and $\square_{i} \eta, i=3,4,5$, but we shall omit the details in this section and $\S \S 3.4,3.5$. We find that

$$
\begin{aligned}
& \square_{3} \xi=\square_{1} \xi, \\
& 2 \bar{\vartheta}_{1} \eta=-\nabla^{k} \eta_{k}+\sqrt{-1}\left(F_{k}^{j} \nabla^{k} \eta_{j}+\eta_{j} \nabla^{k} F_{k}^{j}\right), \\
& 4\left(\partial_{2} \bar{\vartheta}_{1} \eta\right)_{i_{1}}=\eta_{j} F_{i_{1}}{ }^{l} \nabla_{l} \nabla^{k} F_{k}^{j}+F_{i_{1}}{ }^{l} \nabla_{k}{ }^{j} \nabla^{k} \eta_{j}+F_{i_{1}}{ }^{l} \nabla^{k} F_{k}{ }^{j} \nabla_{l} \eta_{j} \\
& -\nabla_{i} \nabla^{k} \eta_{k}+F_{i_{1}}{ }^{l} F_{k}{ }^{j} \nabla_{l} \nabla^{k} \eta_{j}+\sqrt{-1}\left[\eta_{j} \nabla_{i_{1}} \nabla^{k} F_{k}{ }^{j}\right. \\
& \left.+\nabla_{i_{1}} F_{k}^{j} \nabla^{k} \eta_{j}+\nabla^{k} F_{k}^{j} \nabla_{i_{1}} \eta_{j}+F_{k}^{j} \nabla_{i_{1}} \nabla^{k} \eta_{j}+F_{i_{1}}^{l} \nabla_{l} \nabla^{k} \eta_{k}\right] \text {, } \\
& 2\left(\bar{\vartheta}_{1} \partial_{2} \eta\right)_{i_{1}}=2\left[\left(2 \prod_{0,1} \delta \prod_{2,0}+\prod_{1,0} \delta \prod_{2,0}+\prod_{0,1} \delta \prod_{1,1}-\prod_{1,0} \delta \prod_{0,2}\right) \partial_{2} \eta\right]_{i_{1}} \\
& =F_{s}{ }^{j} \nabla^{s} F_{i_{1}}{ }^{k}\left(\nabla_{j} \eta_{k}-\nabla_{k} \eta_{j}\right)+{F_{i}}^{r}\left(\nabla^{s} F_{r}{ }^{j} \nabla_{j} \eta_{s}-\nabla^{s} F_{s}{ }^{j} \nabla_{r} \eta_{j}\right) \\
& -2 \nabla^{s} \nabla_{s} \eta_{i_{1}}+\nabla^{s} \nabla_{i_{1}} \eta_{s}-F_{i_{1}}{ }^{k} F_{s}{ }^{l} \nabla^{s} \nabla_{k} \eta_{l} \\
& +\sqrt{-1}\left[\nabla^{s} F_{i_{1}}{ }^{j}\left(\nabla_{s} \eta_{j}-2 \nabla_{j} \eta_{s}\right)+\nabla^{s} F_{s}{ }^{j}\left(2 \nabla_{j} \eta_{i_{1}}-\nabla_{i_{1}} \eta_{j}\right)\right. \\
& \left.-F_{i_{1}}{ }^{j} \nabla^{s} \nabla_{j} \eta_{s}+F_{s}{ }^{j}\left(2 \nabla^{s} \nabla_{j} \eta_{i_{1}}-\nabla^{s} \nabla_{i_{1}} \eta_{j}\right)\right] \\
& 4\left(\square_{3} \eta\right)_{i_{1}}=\eta_{j} F_{i_{1}}{ }^{l} \nabla_{l} \nabla^{k} F_{k}{ }^{j}+2 F_{s}{ }^{j} \nabla^{s} F_{i_{1}}{ }^{k}\left(\nabla_{j} \eta_{k}-\nabla_{k} \eta_{j}\right) \\
& +F_{i_{1}}{ }^{r}\left(\nabla_{r} F_{k}{ }^{j} \nabla^{k} \eta_{j}-\nabla^{k} F_{k}{ }^{j} \nabla_{r} \eta_{j}\right)+2 F_{i_{1}}{ }^{r} \nabla^{s} F_{r}{ }^{j} \nabla_{j} \eta_{s} \\
& -4 \nabla^{s} \nabla_{s} \eta_{i_{1}}+2 \nabla^{s} \nabla_{i_{1}} \eta_{s}-\nabla_{i_{1}} \nabla^{k} \eta_{k} \\
& +F_{i_{1}}{ }^{l} F_{k}{ }^{j}\left(\nabla_{l} \nabla^{k} \eta_{j}-2 \nabla^{k} \nabla_{l} \eta_{j}\right) \\
& +\sqrt{-1}\left[\eta_{j} \nabla_{i_{1}} \nabla^{k} F_{k}^{j}+\nabla^{s} F_{s}^{j}\left(4 \nabla_{j} \eta_{i_{1}}-\nabla_{i_{1}} \eta_{j}\right)\right. \\
& +2 \nabla^{s} F_{i_{1}}{ }^{j}\left(\nabla_{s} \eta_{j}-2 \nabla_{j} \eta_{s}\right) \\
& +\nabla_{i_{1}} F_{k}{ }^{j} \nabla^{k} \eta_{j}+2 F_{s}{ }^{j}\left(2 \nabla^{s} \nabla_{j} \eta_{i_{1}}-\nabla^{s} \nabla_{i_{1}} \eta_{i}\right) \\
& \left.+F_{i_{1}}{ }^{j}\left(\nabla_{j} \nabla^{k} \eta_{k}-2 \nabla^{k} \nabla_{j} \eta_{k}\right)+F_{k}{ }^{j} \nabla_{i_{1}} \nabla^{k} \eta_{j}\right] \text {. }
\end{aligned}
$$

3.4. Laplacian $\square_{4}$. For $\square_{4} \xi, \square_{4} \eta$ we obtain the following equations:

$$
\begin{gathered}
2\left(\partial_{1} \xi\right)_{i_{1}}=\nabla_{i_{1}} \xi-\sqrt{-1} F_{i_{1}}{ }^{j} \nabla_{j} \xi \\
\square_{4} \xi=\square_{2} \xi \\
2 \bar{\vartheta}_{2} \eta=-\nabla^{k} \eta_{k}+\sqrt{-1} F_{k}{ }^{j} \nabla^{k} \eta_{j}, \\
4\left(\partial_{1} \bar{\vartheta}_{2} \eta\right)_{i_{1}}=F_{i_{1}}{ }^{l} \nabla_{l} F_{k}{ }^{j} \nabla^{k} \eta_{j}-\nabla_{i_{1}} \nabla^{k} \eta_{k}+F_{i_{1}}{ }^{l} F_{k}{ }^{j} \nabla_{l} \nabla^{k} \eta_{j} \\
+\sqrt{-1}\left(\nabla_{i_{1}} F_{k}{ }^{j} \nabla^{k} \eta_{j}+F_{k}{ }^{j} \nabla_{i_{1}} \nabla^{k} \eta_{j}+F_{i_{1}}{ }^{j} \nabla_{j} \nabla^{k} \eta_{k}\right)
\end{gathered}
$$




$$
\begin{aligned}
& -4\left(\bar{\vartheta}_{2} \partial_{1} \eta\right)_{i_{1}}=\eta_{j} F_{k}{ }^{l}\left(\nabla^{k} \nabla_{l} F_{i_{1}}{ }^{j}-\nabla^{k} \nabla_{i_{1}} F_{l}{ }^{j}\right)+\left(\nabla_{l} F_{i_{1}}{ }^{j}-\nabla_{i_{1}} F_{l}{ }^{j}\right) F_{k}{ }^{l} \nabla^{k} \eta_{j} \\
& +F_{l}{ }^{j}\left(\nabla^{l} F_{i_{1}}{ }^{k} \nabla_{k} \eta_{j}-\nabla^{l} F_{j}{ }^{k} \nabla_{k} \eta_{i_{1}}\right)+2 \nabla^{j} \nabla_{j} \eta_{i_{1}}-\nabla^{j} \nabla_{i_{1}} \eta_{j} \\
& +F_{l}{ }^{j} F_{i_{1}}{ }^{k} \nabla^{l} \nabla_{k} \eta_{j}+\sqrt{-1}\left[\left(\nabla^{j} \nabla_{j} F_{i_{1}}{ }^{k}-\nabla^{j} \nabla_{i_{1}} F_{j}{ }^{k}\right) \eta_{k}\right. \\
& +\left(\nabla_{k} F_{i_{1}}{ }^{j}-\nabla_{i_{1}} F_{k}{ }^{j}\right) \nabla^{k} \eta_{j}+\nabla^{j} F_{i_{1}}{ }^{k} \nabla_{k} \eta_{j}-\nabla^{j} F_{j}{ }^{k} \nabla_{k} \eta_{i_{1}} \\
& \left.+F_{i_{1}}{ }^{j} \nabla^{k} \nabla_{j} \eta_{k}-2 F_{j}{ }^{k} \nabla^{j} \nabla_{k} \eta_{i_{1}}+F_{j}{ }^{k} \nabla^{j} \nabla_{i_{1}} \eta_{k}\right] \text {, } \\
& -4\left(\square_{4} \eta\right)_{i_{1}}=\eta_{j} F_{k}{ }^{l}\left(\nabla^{k} \nabla_{l} F_{i_{1}}{ }^{j}-\nabla^{k} \nabla_{i_{1}} F_{l}{ }^{j}\right)+\left(\nabla_{l} F_{i_{1}}{ }^{j}-\nabla_{i_{\mathbf{1}}} F_{l}{ }^{j}\right) F_{k}{ }^{l} \nabla^{k} \eta_{j} \\
& +F_{l}{ }^{j}\left(\nabla^{l} F_{i_{1}}{ }^{k} \nabla_{k} \eta_{j}-\nabla^{l} F_{j}{ }^{k} \nabla_{k} \eta_{i_{1}}\right)-F_{i_{1}}{ }^{l} \nabla_{l} F_{k}{ }^{j} \nabla^{k} \eta_{j} \\
& +\left[\nabla_{i_{1}}, \nabla_{j}\right] \eta^{j}+2 \nabla^{j} \nabla_{j} \eta_{i_{1}}+F_{l}{ }^{j} F_{i_{1}}{ }^{k} \nabla^{l} \nabla_{k} \eta_{j} \\
& -F_{i_{1}}{ }^{l} F_{k}{ }^{j} \nabla_{l} \nabla^{k} \eta_{j} \\
& +\sqrt{-1}\left\{\left(\nabla^{j} \nabla_{j} F_{i_{1}}{ }^{k}-\nabla^{j} \nabla_{i_{1}} F_{j}{ }^{k}\right) \eta_{k}\right. \\
& +\left(\nabla_{k} F_{i_{1}}{ }^{j}-2 \nabla_{i_{1}} F_{k}{ }^{j}\right) \nabla^{k} \eta_{j} \\
& +\nabla^{j} F_{i_{1}}{ }^{k} \nabla_{k} \eta_{j}-\nabla^{j} F_{j}{ }^{k} \nabla_{k} \eta_{i_{1}}+F^{k j}\left[\nabla_{k}, \nabla_{i_{1}}\right] \eta_{j} \\
& \left.+F^{k j}\left[\nabla_{j}, \nabla_{k}\right] \eta_{i_{1}}+F_{i_{1}}{ }^{j}\left[\nabla_{k}, \nabla_{j}\right] \eta^{k}\right\} \text {. }
\end{aligned}
$$

3.5. Laplacian $\square_{5}$. Finally, for the remaining Laplacian $\square_{5}$ we first have

$$
\begin{gathered}
\square_{5} \xi=\square_{1} \xi, \\
\bar{\delta}_{1} \eta=-\eta_{j} \nabla^{i} \prod_{1,0} i^{j}-\prod_{1,0}{ }_{i}^{j} \nabla^{i} \eta_{j} .
\end{gathered}
$$

Adding (3.8) to (3.9) gives

$$
\begin{gathered}
8\left(d_{1} \eta\right)_{i_{1} i_{2}}=4 \nabla_{i_{1}} \eta_{i_{2}}-4 \nabla_{i_{2}} \eta_{i_{1}}+\eta_{j}\left[F_{i_{1}}{ }^{k}\left(\nabla_{i_{2}} F_{k}{ }^{j}-\nabla_{k} F_{i_{2}}{ }^{j}\right)\right. \\
\left.+F_{i_{2}}{ }^{k}\left(\nabla_{k} F_{i_{1}}{ }^{j}-\nabla_{i_{1}} F_{k}{ }^{j}\right)\right] \\
+\sqrt{-1}\left\{\eta _ { j } \left[3 F_{i_{1}}{ }^{k} F_{i_{2}}{ }^{l}\left(\nabla_{k} F_{l}{ }^{j}-\nabla_{l} F_{k}{ }^{j}\right)\right.\right. \\
\left.+\nabla_{i_{1}} F_{i_{2}}{ }^{j}-\nabla_{i_{2}} F_{i_{1}}{ }^{j}\right]+4 F_{i_{2}}{ }^{k} \nabla_{k} \eta_{i_{1}} \\
\left.-4 F_{i_{1}}{ }^{k} \nabla_{k} \eta_{i_{2}}\right\} .
\end{gathered}
$$

Now put

$$
\begin{aligned}
8 G_{i_{1} i_{2}}= & 4 \nabla_{i_{1}} \eta_{i_{2}}+\eta_{j}\left(F_{i_{1}}{ }^{k} \nabla_{i_{2}} F_{k}{ }^{j}+F_{i_{2}}{ }^{k} \nabla_{k} F_{i_{1}}{ }^{j}\right) \\
& +\sqrt{-1}\left(3 \eta_{j} F_{i_{1}}{ }^{k} F_{i_{2}}{ }^{l} \nabla_{k} F_{l}{ }^{j}+\eta_{j} \nabla_{i_{1}} F_{i_{2}}{ }^{j}+4 F_{i_{2}}{ }^{j} \nabla_{j} \eta_{i_{1}}\right) .
\end{aligned}
$$

Then

$$
8 d_{1} \eta=\left(G_{i_{1} i_{2}}-G_{i_{2} i_{1}}\right) d x^{I_{0}(2)} .
$$

As in the derivation of (3.16), (3.17) we can obtain

$$
-2\left(\prod_{0,1} \delta \prod_{1,1} d_{1} \eta\right)_{i_{1}}=\prod_{0,1} \nabla^{s}\left[\left(\varepsilon_{s}^{k_{1}} \varepsilon_{i_{1}}^{k_{2}}-\varepsilon_{i_{1}}^{k_{1}} \varepsilon_{s}^{k_{2}}+F_{s}{ }^{k_{1}}{F_{i_{1}}}^{k_{2}}-F_{i_{1}}{ }^{k_{1}} F_{s}{ }^{k_{2}}\right) G_{i_{1} i_{2}}\right]
$$




$$
-4\left(\prod_{1,0} \delta \prod_{2,0} d_{1} \eta\right)_{i_{1}}=\prod_{1,0} \nabla^{s}\left[\left(B_{s}^{k_{1} k_{1}}+\sqrt{-1} C_{s}^{k_{1} k_{1} k_{2}}\right) G_{k_{1} k_{2}}\right],
$$

where $B_{s i i_{1}}^{k_{1} k_{2}}$ and $C_{s i_{1}}^{k_{1} k_{2}}$ are defined in (3.14). After some calculations we can thus have

$$
\begin{aligned}
16\left(\square_{s} \eta\right)_{i_{1}}= & 16\left[\left(\prod_{1,0} \delta \prod_{2,0}+\prod_{0,1} \delta \prod_{1,1}\right) d_{1} \eta+\prod_{1,0} d \bar{\delta}_{1} \eta\right]_{i_{1}} \\
= & \eta_{j}\left[\nabla^{s} F_{i_{1}}{ }^{k}\left(\nabla_{s} F_{k}{ }^{j}-\nabla_{k} F_{s}{ }^{j}\right)+F_{i_{1}}{ }^{r} F_{s}{ }^{k} \nabla^{s} F_{r}{ }^{l}\left(\nabla_{k} F_{l}{ }^{j}\right.\right. \\
& \left.\left.-\nabla_{l} F_{k}{ }^{j}\right)+4 F_{i_{1}}{ }^{k}\left(\nabla^{s} \nabla_{s} F_{k}{ }^{j}-\nabla^{s} \nabla_{k} F_{s}{ }^{j}+\nabla_{k} \nabla^{l} F_{l}{ }^{j}\right)\right] \\
& +8 F_{i_{1}}{ }^{k} \nabla_{s} F_{k} F_{k}^{j} \nabla^{s} \eta_{j}-8 \nabla^{s} \nabla_{s} \eta_{i_{1}}+4\left[\nabla_{k}, \nabla_{i_{1}}\right] \eta^{k} \\
& +4 F_{i_{1}}{ }^{k} F^{k j}\left[\nabla_{l}, \nabla_{k}\right] \eta_{j}+\sqrt{-1}\left\{\eta _ { j } \left[4 F _ { i _ { 1 } } { } ^ { k } \nabla ^ { s } F _ { s } { } ^ { l } \left(\nabla_{k} F_{l}{ }^{j}\right.\right.\right. \\
& \left.-\nabla_{l} F_{k}{ }^{j}\right)+F_{i_{1}}{ }^{r} \nabla^{s} F_{r}{ }^{k}\left(\nabla_{k} F_{s}{ }^{j}-\nabla_{s} F_{k}{ }^{j}\right) \\
& +3 F_{s}{ }^{k} \nabla^{s} F_{i_{1}}{ }^{l}\left(\nabla_{l} F_{k}{ }^{j}-\nabla_{k} F_{l}{ }^{j}\right)+4 \nabla_{i_{1}} \nabla^{k} F_{k}{ }^{j} \\
& \left.+4 F_{i_{1}}{ }^{k} F_{s}{ }^{l} \nabla^{s}\left(\nabla_{k} F_{l}{ }^{j}-\nabla_{l} F_{k}{ }^{j}\right)\right]+8 \nabla^{s} F_{s}{ }^{k} \nabla_{k} \eta_{i_{1}} \\
& +4 F_{i_{1}}{ }^{r} F^{s k}\left(\nabla_{r} F_{s}{ }^{l}+\nabla_{s} F_{r}{ }^{l}\right) \nabla_{l} \eta_{k}-4\left(\nabla_{i_{1}} F_{j}{ }^{k}+\nabla_{j} F_{i_{1}}{ }^{k}\right) \nabla_{k} \eta^{j} \\
& \left.+4 F^{k j}\left[\nabla_{i_{1}}, \nabla_{k}\right] \eta_{j}+4 F_{i_{1}}{ }^{l}\left[\nabla_{l}, \nabla_{k}\right] \eta^{k}+4 F^{s k}\left[\nabla_{s}, \nabla_{k}\right] \eta_{i_{1}}\right\}
\end{aligned}
$$

\section{Realization of $\square$ 's}

Theorem 4.1. The complex Laplacian $\square_{i}, i=1, \ldots, 5$, for an almostHermitian structure is real with respect to every 0 -form if and only if the structure is almost-semi-Kählerian. Moreover, with respect to every 0-form, if $\square_{i}$, $i=1, \ldots, 5$, for an almost-Hermitian structure is real, then $\square_{i}=\Delta / 2$ for $i=1, \ldots, 5$.

Proof. The theorem follows immediately from (3.1), (3.5), (3.22), (3.28), (3.33) and (2.8) by choosing the 0 -form $\xi$ to be $x^{k}$ for an arbitary $k$ with respect to any local coordinates $x^{1}, \cdots, x^{n}$.

Theorem 4.2. For an almost-Hermitian structure, if the Laplacian $\square_{i}$, $i=1,2$ or 4 , is real with respect to all 0 - and 1-forms, then the structure is Kählerian.

Kodaira and Spencer [3] have shown that if the relation

$$
\square_{1}=\Delta / 2
$$

holds for an almost-Hermitian structure, then the structure is integrable. The particular case of Theorem 4.2 in which

$$
\square_{i}=\Delta / 2 \quad(i=1,2 \text { or } 4)
$$

holds was a conjecture for some time; it was proved by Hsiung [2] for $i=2$ and by A. W. Adler [1] for $i=1$ by a different method under a stronger as- 
sumption that (4.1) holds for a Hermitian structure and all 0-, 1- and 2-forms. Theorem 4.2 was proved by Hsiung [2] and Ogawa [5] for $i=2$, and by Ogawa [5] for $i=1$ by a somewhat different method.

Proof. (i) $i=2$. In [2, p. 148] Hsiung proved that under the assumption of the theorem the structure $F$ satisfies $^{2}(2.17)$ and (2.28). Then the theorem follows immediately from Lemmas 2.4 and 2.3; this was pointed out to one of the authors by $\mathrm{H}$. Wakakuwa.

(ii) $i=1$. Using the Ricci and Bianchi identities and (2.23) we can easily obtain

$$
\begin{aligned}
& F_{i_{1}}{ }^{k}\left[\nabla_{j}, \nabla_{k}\right] \eta^{j}=F_{i_{1}}{ }^{k} R_{k}{ }^{j} \eta_{j}, \\
& F^{k j}\left[\nabla_{k}, \nabla_{i_{1}}\right] \eta_{j}-F^{j k}\left[\nabla_{j}, \nabla_{k}\right] \eta_{i_{1}}=-\frac{1}{2} F^{k l} R^{j}{ }_{i_{1} k l} \eta_{j}, \\
& \nabla_{s} \nabla_{i_{1}} F^{s j}-\nabla_{i_{1}} \nabla_{s} F^{s j}=F_{a}{ }^{j} R_{i_{1}}{ }^{a}-\frac{1}{2} F^{s a} R^{j}{ }_{i_{1} s a} .
\end{aligned}
$$

By assumption, for any 1 -form $\eta, \operatorname{Im} \square_{1} \eta=0$ which is reduced to, in consequence of Theorem 4.1, (2.8), (3.21), (4.3), (4.4), (4.5),

$$
2\left(\nabla_{s} F_{i_{1}}{ }^{j}+\nabla_{i_{1}} F_{s}{ }^{j}\right) \nabla_{j} \eta^{s}+\left(\nabla^{s} \nabla_{s} F_{i_{1}}{ }^{j}+F_{i_{1}}{ }^{k} R_{k}{ }^{j}-R_{i_{1}}{ }^{k} F_{k}{ }^{j}\right) \eta_{j}=0
$$

By choosing

$$
\eta=d x^{h}, \quad \text { for an arbitary } h
$$

with respect to any local coordinates $x^{1}, \cdots, x^{n}$, from (4.6) it thus follows that

$$
\nabla^{s} \nabla_{s} F_{i_{1}}{ }^{h}+F_{i_{1}}{ }^{k} R_{k}{ }^{h}-R_{i_{1}}{ }^{k} F_{k}{ }^{h}=0 .
$$

Multiplying (4.8) by $F_{h}{ }^{i_{1}}$ and using (2.1) we obtain (2.16), and therefore the structure $F$ is Kählerian by Lemma 2.2 .

(iii) $\quad i=4$. At a general point $P$ of the manifold $M^{n}$ we choose orthogonal geodesic local coordinates $x^{1}, \cdots, x^{n}$ so that

$$
g_{i j}(P)=\delta_{i j}, \quad \Gamma_{i j}^{k}(P)=0,
$$

where $\delta_{i j}$ are Kronecker deltas. By using Theorem 4.1, and choosing $\eta$ to satisfy (4.7) first and then

$$
\eta=x^{h} d x^{l}, \quad \text { for any fixed distinct } h \text { and } l
$$

with respect to the geodesic local coordinates $x^{1}, \cdots, x^{n}$, from (3.32) the condition $\operatorname{Im}\left(\square_{4} \eta\right)=0$ for any 1 -form $\eta$ is reduced to

$$
\nabla^{j} \nabla_{j} F_{i_{1}}{ }^{h}-\nabla^{j} \nabla_{i_{1}} F_{j}{ }^{h}=0
$$

\footnotetext{
${ }^{2}$ By mistake, (2.28) was printed as $F_{i}{ }^{h} R^{i}{ }_{j k l}=F_{j}{ }^{i} R^{h}{ }_{i k l}$ in [2, p. 148].0
} 


$$
\nabla^{h} F_{i_{1} l}+2 \nabla_{i_{1}} F_{l}{ }^{h}+\nabla_{l} F_{i_{1}}{ }^{h}=0 .
$$

Interchanging $l, i_{1}$ in (4.12) and adding the resulting equation to (4.12) we obtain

$$
\nabla_{i_{\mathbf{1}}} F_{l}{ }^{h}+\nabla_{l} F_{i_{1}}{ }^{h}=0 .
$$

From (4.11), (4.13) it thus follows that

$$
\nabla^{j} \nabla_{j} F_{i_{1}}{ }^{h}=0,
$$

and hence by Lemma 2.2 the structure is Kählerian.

\section{Relationships among $\square$ 's}

Theorem 5.1. If for an almost-Hermitian structure the relation

$$
\operatorname{Im} \square_{1}=\operatorname{Im} \square_{i} \quad(i=2 \text { or } 4)
$$

holds for all 0- and 1-forms, then the structure is Kählerian.

Proof. (i) $i=2$. From (3.5), (3.1) and condition (5.1) for any 0-form $\xi$, we have

$$
\nabla^{h} F_{h}{ }^{j} \nabla_{j} \xi=0 .
$$

By choosing $\xi=x^{i}$ for an arbitary $i$ with respect to any local coordinates $x^{1}$, $\cdots, x^{n}$, from (5.2) follows immediately (2.8), which together with (3.2), (3.21), (3.20) reduces condition (5.1) for any 1 -form $\eta$ to

$$
\left(\nabla_{s}{F_{i_{1}}}^{j}+\nabla_{i_{1}} F_{s}{ }^{j}\right) \nabla_{j} \eta^{s}-\left(\nabla^{s} \nabla_{i_{1}} F_{s}{ }^{j}-\nabla^{s} \nabla_{s} F_{i_{1}}{ }^{j}\right) \eta_{j}=0 .
$$

Choosing $\eta$ to satisfy (4.7) first and then (4.10) with respect to the local coordinates $x^{1}, \cdots, x^{n}$ defined by (4.9) we therefore obtain (4.11), (4.13), and hence the structure is Kählerian for the same reasoning given in the proof (iii) of Theorem 4.2 .

(ii) $i=4$. As in part (i), from (3.5), (3.28), (3.1) and condition (5.1) for any 0 -form $\xi$, we obtain (2.8), which together with (3.21), (3.32) reduces condition (5.1) for any 1-form $\eta$ to

$$
\left(\nabla_{j} F_{i_{1}}{ }^{k}-\nabla^{k} F_{i_{1} j}\right) \nabla_{k} \eta^{j}=0 .
$$

By choosing $\eta$ to satisfy (4.10) with respect to the local coordinates $x^{1}, \cdots, x^{n}$ defined by (4.9), we have

$$
\nabla_{l} F_{h i_{1}}-\nabla_{h} F_{l i_{1}}=0
$$

Thus by Lemma 2.1 the structure is Kählerian.

Theorem 5.2. If for an almost-Hermitian structure either the relation 


$$
\operatorname{Im} \square_{2}=\operatorname{Im} \square_{4}
$$

or

$$
\operatorname{Re} \square_{2}=\operatorname{Re} \square_{4}
$$

holds for all 1-forms, where $\mathrm{Re}$ denotes the real part, then the structure is Kählerian.

Proof. From (3.1), (3.32), by the same argument as in the proof of Theorem 5.1 for $i=4$ it is easily seen that conditions (5.6), (5.7) imply

$$
\begin{gathered}
\nabla_{h} F_{i_{1}}{ }^{l}=\nabla_{i_{1}} F_{h}{ }^{l}=0, \\
F_{h}{ }^{j} \nabla_{i_{1}} F_{j}{ }^{l}-F_{h}{ }^{j} \nabla_{j} F_{i_{1}}{ }^{l}=0,
\end{gathered}
$$

respectively. By multiplying (5.9) by $F_{k}{ }^{h}$, we can reduce (5.9) to (5.8). Hence by Lemma 2.1, the structure is Kählerian under either (5.6) or (5.7).

Theorem 5.3. If for an almost-Hermitian structure the relation

$$
\operatorname{Im} \square_{2}=\operatorname{Im} \square_{5}
$$

holds for all 0- and 1-forms, then the structure is Kählerian.

Proof. From (3.33), (3.5), (3.1) and condition (5.10) for any 0-form $\xi$ we obtain (2.8). Then by the same argument as in the proof of Therem 5.1 for $i=2,(2.8),(3.2),(3.40)$ reduce condition (5.10) for any 1 -form $\eta$ to

$$
\begin{gathered}
F_{i_{1}}{ }^{r} \nabla^{s} F_{r}{ }^{k}\left(\nabla_{k} F_{s}{ }^{h}-\nabla_{s} F_{k}{ }^{h}\right)+3 F_{s}{ }^{k} \nabla^{s} F_{i_{1}}{ }^{l}\left(\nabla_{l} F_{k}{ }^{h}-\nabla_{k} F_{l}{ }^{h}\right) \\
+4 F_{i_{1}}{ }^{k} F_{s}{ }^{l} \nabla^{s}\left(\nabla_{k} F_{l}{ }^{h}-\nabla_{l} F_{k}{ }^{h}\right)=0 \\
F_{i_{1}}{ }^{r} F^{s l}\left(\nabla_{r} F_{s}{ }^{h}+\nabla_{s} F_{r}{ }^{h}\right)=0 .
\end{gathered}
$$

Multiplying (5.12) by $F_{j}{ }^{i_{1}} F_{l}{ }^{k}$ and use of (2.1) give

$$
\nabla_{j} F_{k}{ }^{h}+\nabla_{k} F_{j}{ }^{h}=0 \text {. }
$$

Substituting (5.13) in (5.11) we can easily obtain

$$
2 F_{i_{1}}{ }^{k} F_{s}{ }^{l} \nabla^{s} \nabla_{k} F_{l}{ }^{h}-F_{s}{ }^{k} \nabla^{s} F_{i_{1}}{ }^{l} \nabla_{k} F_{l}{ }^{h}=0 .
$$

Multiplying (5.14) by $F_{h}^{i_{1}}$ and using (2.1), (2.8), (5.13) we therefore have

$$
\nabla_{s} F_{i_{1} l} \nabla^{s} F^{i_{1} l}=0,
$$

which implies that $\nabla_{s} F_{i_{1} l}=0$. Hence the structure is Kählerian. q.e.d.

Finally, it should be remarked that there are no theorems involving the Laplacian $\square_{3}$ similar to Theorems 4.2, 5.1, 5.2, 5.3. However, we have the following two theorems, the proofs of which are omitted. 
Theorem 5.4. If for an almost-Hermitian struciure the relation

$$
\operatorname{Im} \square_{3}=\operatorname{Im} \square_{1}+\frac{1}{2} \operatorname{Im}\left(\bar{\vartheta}_{1} \partial_{2}\right)
$$

holds for all 1-forms, then the structure is Kählerian.

Theorem 5.5. If for an almost-semi-Kählerian structure the relation

$$
\operatorname{Im} \square_{3}=\operatorname{Im} \square_{i}+\frac{1}{2} \operatorname{Im}\left(\bar{\vartheta}_{1} \partial_{2}\right) \quad(i=2 \text { or } 4)
$$

holds for all 1-forms, then the structure is Kählerian.

\section{References}

[1] A. W. Adler, Classifying spaces for Kähler metrics IV: The relation $\Delta=2 \cdot \square$, Math. Ann. 160 (1965) 41-58.

[2] C. C. Hsiung, Structures and operators on almost-Hermitian manifolds, Trans. Amer. Math. Soc. 122 (1966) 136-152.

[ 3 ] K. Kodaira \& D. C. Spencer, On the variation of almost-complex structure, Algebraic Geometry and Topology, A Symposium in Honor of S. Lefschetz, Princeton University Press, Princeton, 1957, 139-150.

[ 4 ] S. Kotô, Some theorems on almost Kählerian spaces, J. Math. Soc. Japan 12 (1960) $422-433$.

[ 5] Y. Ogawa, Operators on almost Hermitian manifolds, J. Differential Geometry 4 (1970) 105-119.

[6] G. de Rham \& K. Kodaira, Harmonic integrals, Mimeographed notes, Institute for Advanced Study, Princeton, 1950.

[7] M. Schiffer \& D. C. Spencer, Functionals of finite Riemann surfaces, Princeton University Press, Princeton, 1954.

LEHIGH UNIVERSITY 
